\title{
El Extracto Acuoso de Orégano (Lippia graveolens HBK) del Norte de México Tiene Actividad Antioxidante sin Mostrar un Efecto Tóxico in vitro $\mathrm{e}$ in vivo
}

\author{
The Aqueous Extract from Oregano (Lippia graveolens HBK) from the North of Mexico \\ Has Antioxidant Activity without Showing a Toxic Effect in vitro and in vivo
}

\author{
"Adolfo Soto-Domínguez; "Rubén García-Garza; ** Yolanda Ramírez-Casas; \\ ${ }^{* * * *}$ Javier Morán-Martínez \& ** Luis Benjamín Serrano-Gallardo
}

SOTO-DOMÍNGUEZ, A.; GARCÍA-GARZA, R.; RAMÍREZ-CASAS, Y.; MORÁN-MARTÍNEZ, J. \& SERRANO-GALLARDO, L. B. El extracto acuoso de oregano (Lippia graveolens HBK) del norte de México tiene actividad antioxidante sin mostrar un efecto toxico in vitro e in vivo. Int. J. Morphol., 30(3):937-944, 2012.

RESUMEN: Desde tiempos antiguos la medicina tradicional ha usado extensamente las especies del género Lippia como analgésicos, antiinflamatorios, antipiréticos, antifúngicos, etc. Numerosos estudios describen diversos compuestos presentes en extractos acuosos, metanólicos, o aceites esenciales de estas plantas, siendo los flavonoides los compuestos más abundantes. Sin embargo, la composición y cantidad de los metabolitos secundarios dependen de la zona geográfica, factores climáticos, altitud, época de cosecha y estado de crecimiento de estas plantas. El objetivo de este estudio fue evaluar la actividad antioxidante del extracto acuoso de orégano (Lippia graveolens HBK) del Norte de México y su efecto tóxico in vitro e in vivo. La capacidad antioxidante del extracto acuoso se midió por el método de DPPH en seis diluciones del extracto $(5-160 \mathrm{mg} / \mathrm{mL})$ y se utilizó Trolox como referencia; para el efecto tóxico in vitro se usó el ensayo de citotoxicidad con larvas de Artemia salina. Para el efecto in vivo se emplearon 24 ratones árabes machos divididos en 6 grupos de animales (n=4): 4 grupos experimentales con 10, 100, 1000 y $5000 \mathrm{mg}$ del extracto/ kg de peso administrados vía oral respectivamente, además de un grupo control positivo ( $5 \mathrm{mg}$ de colchicina/ $\mathrm{kg}$ de peso vía i.p) y un grupo control negativo (solo agua destilada). Después del tratamiento los ratones se sacrificaron y se colectaron muestras de hígado y riñón que se sometieron a estudios histológicos e histoquímicos, además se realizó un análisis cuantitativo. La actividad antioxidante del extracto acuoso de orégano se presentó a $160 \mathrm{mg} / \mathrm{mL}$. La CL50 fue mayor a 1,000 $\mu \mathrm{g} / \mathrm{mL}$ por lo que el extracto se considera no tóxico. En el análisis morfológico in vivo con distintas dosis del extracto acuoso de orégano no se observó un efecto tóxico. Los resultados obtenidos validan el uso del orégano en la medicina tradicional.

PALABRAS CLAVE: Antioxidante; Orégano; Análisis morfológico.

\section{INTRODUCCIÓN}

El nombre orégano comprende más de veinticuatro diferentes especies de plantas, entre las que destacan el orégano europeo Origanum vulgare de la familia Lamiaceae, y el orégano mexicano Lippia graveolens perteneciente a la familia Verbenacea (Rzedowski, 1978; Huerta, 1997; Pierce, 1999). Desde tiempos antiguos la medicina tradicional ha usado diversas especies del género Lippia como analgésicos, antiinflamatorios, antipiréticos; contra diarrea, vómito, indigestión y disentería (Monroy \& Castillo, 2000); como antialérgicos, antivirales, vasodilatadores y antihipertensivos (Pietta, 1999; Guerrero et al., 2002), antifúngicos (Hernández et al., 2008), antiparasitarios (Bassole et al., 2003); antígenotóxicos (Sivropoulous et al., 1996; He et al., 1997; Hirobe et al., 1998); antimicrobiano en infecciones cutáneas, gástricas y respiratorias (Pascual et al., 2001; Skandamis et al., 2002; Burt \& Reinders, 2003; Hernández et al., 2009); espasmolíticos y estrogénicos (Craker \& Simon, 1992; de las Heras et al., 1998; Pérez, 2002); en el tratamiento de desórdenes hepáticos, diurético, remedio de desórdenes menstruales, repelente, antimalaria, tratamiento contra sífilis, gonorrea y diabetes (Socorro et al., 1998; Pascual et al.; Arcila-Lozano et al., 2004).

\footnotetext{
* Departamento de Histología, Facultad de Medicina. Universidad Autónoma de Nuevo León, México.

** Departamento de Bioquímica y Farmacología, Centro de Investigación Biomédica, Universidad Autónoma de Coahuila, México.

**** Departamento de Biología Celular y Ultraestructura, Centro de Investigación Biomédica, Universidad Autónoma de Coahuila, México.
} 
SOTO-DOMínGUEZ, A.; GARCÍA-GARZA, R.; RAMíREZ-CASAS, Y.; MORÁN-MARTíNEZ, J. \& SERRANO-GALLARDO, L. B. El extracto acuoso de oregano (Lippia graveolens HBK) del norte de México tiene actividad antioxidante sin mostrar un efecto toxico in vitro e in vivo. Int. J. Morphol., 30(3):937-944, 2012.

A la fecha existen numerosos estudios en los que se han identificado una gran cantidad de compuestos químicos que varían de acuerdo a la variedad de Lippia analizada empleando extractos acuosos (Valentão et al., 2002; Ricco et al., 2010), metanólicos y etanólicos (Zétola et al., 2002; González-Güereca et al., 2007; Salinas-Sánchez et al., 2009) y aceites esenciales de las hojas y tallos (Pascual et al.; Bassole et al.; Salgueiro et al., 2003, Hernández et al., 2008, 2009). Siendo los flavonoides los compuestos antioxidantes más abundantes en Lippia graveolens (Rzedowski; Pietta; Dragland et al., 2003). Sin embargo la composición y cantidad de los metabolitos secundarios de estas plantas dependen de la zona geográfica, factores climáticos, altitud, época de cosecha y su estado de crecimiento (Cimanga et al., 2002; Lecona-Uribe et al., 2003; Salgueiro et al.; Hernández et al., 2009), por lo que el objetivo de este estudio fue el evaluar la actividad antioxidante del extracto acuoso de orégano (Lippia graveolens HBK) del norte de México y su efecto tóxico in vitro e in vivo.

\section{MATERIAL Y MÉTODO}

Recolección del material vegetal y procesamiento para obtener el extracto acuoso. Se recolectó material vegetal fresco entre los meses de mayo-agosto del 2008 en la Sierra de Jimulco, ubicada a 1,200 metros sobre el nivel del mar en el Municipio de Torreón, Coahuila, al Norte de México. Las plantas se colectaron antes de la floración y un ejemplar fue identificado por el M.C. Eduardo Blanco Contreras, de la Universidad Autónoma Agraria Antonio Narro, Unidad Laguna. El material se secó a la sombra a temperatura ambiente (TA), se separaron las hojas del tallo, y se trituraron hasta obtener un polvo fino que se pasó por una malla 20. El extracto acuoso se preparó por extracción en ebullición de una mezcla del polvo en agua a $10 \%(\mathrm{~m} / \mathrm{v})$ durante 10 min. El extracto se filtró a vacío, se llevó a sequedad total en una estufa a $37^{\circ} \mathrm{C}$ y se almacenó en frasco de vidrio ámbar a $4^{\circ} \mathrm{C}$ hasta su estudio. A partir de este polvo se realizaron las diluciones.

Determinación de la actividad antioxidante por el método de DPPH. Como indicador de la actividad antioxidante se siguió el procedimiento descrito por Ohinishi et al. (1994), en donde el radical libre 2,2,'-difenil-1-picrilhidracilo (DPPH) es reducido a 2,2,'-difenil-1-picril hidracina por compuestos que contienen grupos - $\mathrm{OH}$ que decoloran el reactivo. Se realizaron seis diluciones $(5-160 \mathrm{mg} / \mathrm{mL})$ del polvo en agua destilada, en seguida se tomaron $3 \mathrm{~mL}$ de cada dilución y se adicionó $1 \mathrm{~mL}$ de la solución de DPPH $0,1 \mathrm{mM}$ disuelto en metanol al $80 \%$, en seguida se midieron las absorbancias a 0 y $30 \mathrm{~min}(\mathrm{Abst}=0$ y Abst=30 min res- pectivamente) a $517 \mathrm{~nm}$ con un espectrofotómetro marca Spectronic 20 Genesys. La curva de calibración se realizó empleando diluciones crecientes de Trolox ${ }^{\circledR}$ (6-hidroxi2,5,7,8-tetrametilcromo-2-ácido carboxílico 97\%, Sigma Aldrich Chemical Co., Gillingham, Dorset, UK) en metanol al $80 \%$ como antioxidante de referencia para comparar la actividad antioxidante del extracto.

Ensayo de toxicidad in vitro con larvas de Artemia salina. Se pesaron $40 \mathrm{mg}$ de huevos de Artemia salina (Brine Shrimp Eggs® de Salt Creek Inc.) y se depositaron en 200 $\mathrm{mL}$ de agua de mar artificial (Instant Ocean®) a pH 8 y densidad de 1.020-1.025 y se incubaron a TA por $48 \mathrm{~h}$ bajo una lámpara de luz blanca hasta eclosión de los huevos a larvas. Posteriormente se tomaron 10 larvas de Artemia salina y se depositaron en tubos de ensayo que contenían 5 $\mathrm{mL}$ de las soluciones de 10, 50, 100, 500 y $1000 \mu \mathrm{g} / \mathrm{mL}$ de concentración del polvo seco en agua de mar destilada y se incubaron por $24 \mathrm{~h}$ a $\sim 40^{\circ} \mathrm{C}$. Se contaron las larvas vivas y muertas con ayuda de un microscopio estereoscópico y los datos se analizaron con el paquete estadístico de Finney (Análsis Probit) para la determinación de la concentración letal 50 (CL50) con un 95\% de intervalo de confianza, esto se realizó por triplicado. Con el ensayo de toxicidad para larvas de Artemia salina, se considera que los compuestos biológicamente activos son aquellos con una concentración letal media (CL50) de 0 a $5 \mathrm{mg} / \mathrm{mL}$ como altamente tóxica, de 6 a $200 \mathrm{mg} / \mathrm{mL}$ como tóxica, y no tóxica 200 a $1000 \mathrm{mg} /$ $\mathrm{mL}$ (Meyer et al., 1982).

Evaluación in vivo del extracto acuoso de Lippia graveolens HBK. Se emplearon 24 ratones árabes machos, mantenidos en condiciones estándares del bioterio, con humedad, ciclos de luz y oscuridad, agua y comida ad libitum. Se trabajó con 6 grupos de animales $(n=4)$ : 4 grupos experimentales con 10, 100, 1000 y $5000 \mathrm{mg}$ del extracto/ kg de peso administrados vía oral respectivamente, para determinar las dosis administradas in vivo se tomó como base la CL50 calculada de acuerdo a los resultados de Artemia salina. Al grupo control positivo se le administró una dosis de 5 $\mathrm{mg}$ de colchicina/kg de peso vía i.p. y al grupo control negativo (solo agua destilada vía oral).

Los ratones de todos los grupos fueron sacrificados por dislocación cervical según las Normas de la Convención de Helsinki para la experimentación a 24 hrs posteriores a la administración del extracto, colchicina o agua. Después del sacrificio, todos los animales se perfundieron a través del ventrículo izquierdo con una solución fijadora de formalina al $10 \%$ en solución buffer de fosfatos (PBS) 1X, $\mathrm{pH}$ 7.2-7.4. En seguida se disecaron muestras de hígado y riñón que fueron procesadas por la técnica histológica hasta su inclusión definitiva en bloques de parafina, se obtuvie- 
SOTO-DOMÍNGUEZ, A.; GARCÍA-GARZA, R.; RAMÍREZ-CASAS, Y.; MORÁN-MARTÍNEZ, J. \& SERRANO-GALLARDO, L. B. El extracto acuoso de oregano (Lippia graveolens HBK) del norte de México tiene actividad antioxidante sin mostrar un efecto toxico in vitro e in vivo. Int. J. Morphol., 30(3):937-944, 2012.

ron cortes histológicos de $5 \mu \mathrm{m}$ de grosor teñidos con Hematoxilina y Eosina ( $\mathrm{H}$ y E), tricrómico de Masson e histoquímica con el ácido peryódico de Shiff (PAS). Estos cortes se analizaron mediante microscopia de luz para su evaluación morfológica comparando los grupos experimentales con los grupos control negativo y control positivo en un estudio ciego donde participaron tres especialistas en morfología. También se realizó un análisis cuantitativo en donde el parénquima del hígado se dividió en acinos hepáticos o de Rappaport diferenciando las 3 zonas descritas para este, se cuantificaron los hepatocitos normales diferenciándolos de los hepatocitos con signos de necrosis. Debido a que en el riñón un efecto tóxico se evidencia por daño en los túbulos contorneados proximales y distales, en este estudio se realizó un conteo de estas estructuras con o sin signos de alteración en su histología. Para ambos órganos de los grupos de estudio se analizaron 8 campos consecutivos, esto se realizó por triplicado. De los valores porcentuales obtenidos se obtuvieron valor promedio y desviación estándar (DE) y se analizaron estadísticamente con la prueba de T-student para un valor de significancia $\mathrm{p} \leq 0.05$.

\section{RESULTADOS Y DISCUSION}

Actividad antioxidante. Los resultados de nuestro estudio indican que el orégano Lippia graveolens HBK de la sierra de Jimulco, en el estado de Coahuila del Norte de México, presenta actividad antioxidante que se incrementa conforme aumentan las concentraciones del extracto sin tener un efecto toxico in vitro e in vivo. La mayor actividad antioxidante se presenta a $160 \mathrm{mg} / \mathrm{mL}$, esta concentración es menor a las descritas en el estudio de Zavala-Nigoa et al. (2006) usando extractos acuosos de orégano (Lippia graveolens Kunt) de los estados de Guanajuato, Querétaro y Puebla, México, en donde se encontraron valores de la capacidad antioxidante a 357,15 $\pm 11,84,397,99 \pm 22,02$ y 406,96 $\pm 15,14 \mathrm{mg} / \mathrm{ml}$, respectivamente. Esta concentración además es mucho mayor a la descrita por González-Güereca et al. (500 mg/L) en el 2007, usando un extracto acuoso del tallo Lippia graveolens HBK var. berlandieri Schauer, del estado de Durango, México. Esta variación en las concentraciones del efecto antioxidante observado entre plantas de distintos estados en el territorio nacional puede ser debido a que Lippia graveolens presenta una composición y cantidad de los metabolitos secundarios que dependen de la zona geográfica, factores climáticos, altitud, época de cosecha y su estado de crecimiento (Cimanga et al.; Lecona-Uribe et al.; Salgueiro et al., Hernández et al., 2009), por lo que es nuestros resultados confirman que la cantidad de compuestos antioxidantes presentes en las plantas del género Lippia es distinta en cada una de las regiones.
Efecto tóxico in vitro (CL50). Los resultados de la concentración letal media (CL50) para evaluar el efecto toxico in vitro del extracto fue mayor a 1,000 $\mu \mathrm{g} / \mathrm{mL}(354428.84 \mu \mathrm{g} /$ $\mathrm{mL}$ ), por lo que el extracto acuoso de Lippia graveolens se considera no tóxico, tomando en cuenta el criterio de que una $\mathrm{DL} 50=0-5 \mathrm{mg} / \mathrm{mL}$ es altamente tóxica, una DL50 de 6 - 200mg/mL es tóxica, y una DL50 de 200 - $1000 \mathrm{mg} / \mathrm{mL}$ no es tóxica. Esta prueba in vitro de toxicidad con larvas de Artemia salina se considera como una prueba general para analizar los extractos de plantas (Meyer et al.). La viabilidad de las larvas de Artemia salina en los tubos sin extracto fue del $100 \%$.

Efecto tóxico in vivo y análisis cuantitativo. En el análisis morfológico de daño tisular in vivo del extracto acuoso de Lippia graveolens HBK sobre el hígado y riñón de ratones tratados a distintas concentraciones, no se observó un efecto tóxico sobre las células de cada una de las regiones de estos órganos. En el hígado de los ratones del grupo control negativo se observaron los hepatocitos con límites celulares bien definidos y estructura de los lobulillos hepáticos con una apariencia histológica normal (Fig. 1 A y B). Con la técnica histoquímica de PAS, se observaron hepatocitos con material positivo que corresponde a depósitos de glucógeno de aspecto granulado (Fig. $1 \mathrm{C}$ ). En los ratones tratados con colchicina, se observó daño generalizado en los hepatocitos, incremento en su tamaño, presencia de abundantes vacuolas en su citoplasma, signos de necrosis y alteración de la arquitectura normal caracterizada por desorganización de las láminas de hepatocitos, dilatación de los capilares sinusoidales y zonas de hemorragia (Fig. 1 D y E). Con la técnica de PAS se observaron amplias zonas de células carentes de positividad por la pérdida de glucógeno alternando con zonas con material PAS positivo en el espacio extracelular (Fig. 1 F). En los animales tratados con el extracto acuso de orégano se observó una histología normal para éste órgano similar a la observada en el grupo control negativo (Fig. $1 \mathrm{G}, \mathrm{H} \mathrm{e} \mathrm{I}$ ).

En el análisis cuantitativo del hígado en el grupo control positivo se encontró una considerable disminución en el porcentaje de hepatocitos normales en las 3 zonas de estudio sin encontrar diferencias significativas entre las mismas $(65,41 \pm 4,58)$, los valores del grupo control negativo fueron de $99,91 \pm 0,12$. Cabe destacar que en los grupos tratados con el extracto de orégano, a la mayor concentración (5000 $\mathrm{mg} / \mathrm{kg}$ de peso) los valores fueron de 99,08 $\pm 1,02$ sin observar diferencia estadística significativas en el porcentaje de hepatocitos normales de las 3 zonas de estudio (Tabla I).

En la corteza y médula de riñón del grupo control negativo se observaron corpúsculos renales, túbulos contorneados proximales, distales, asas de Henle y túbulos 


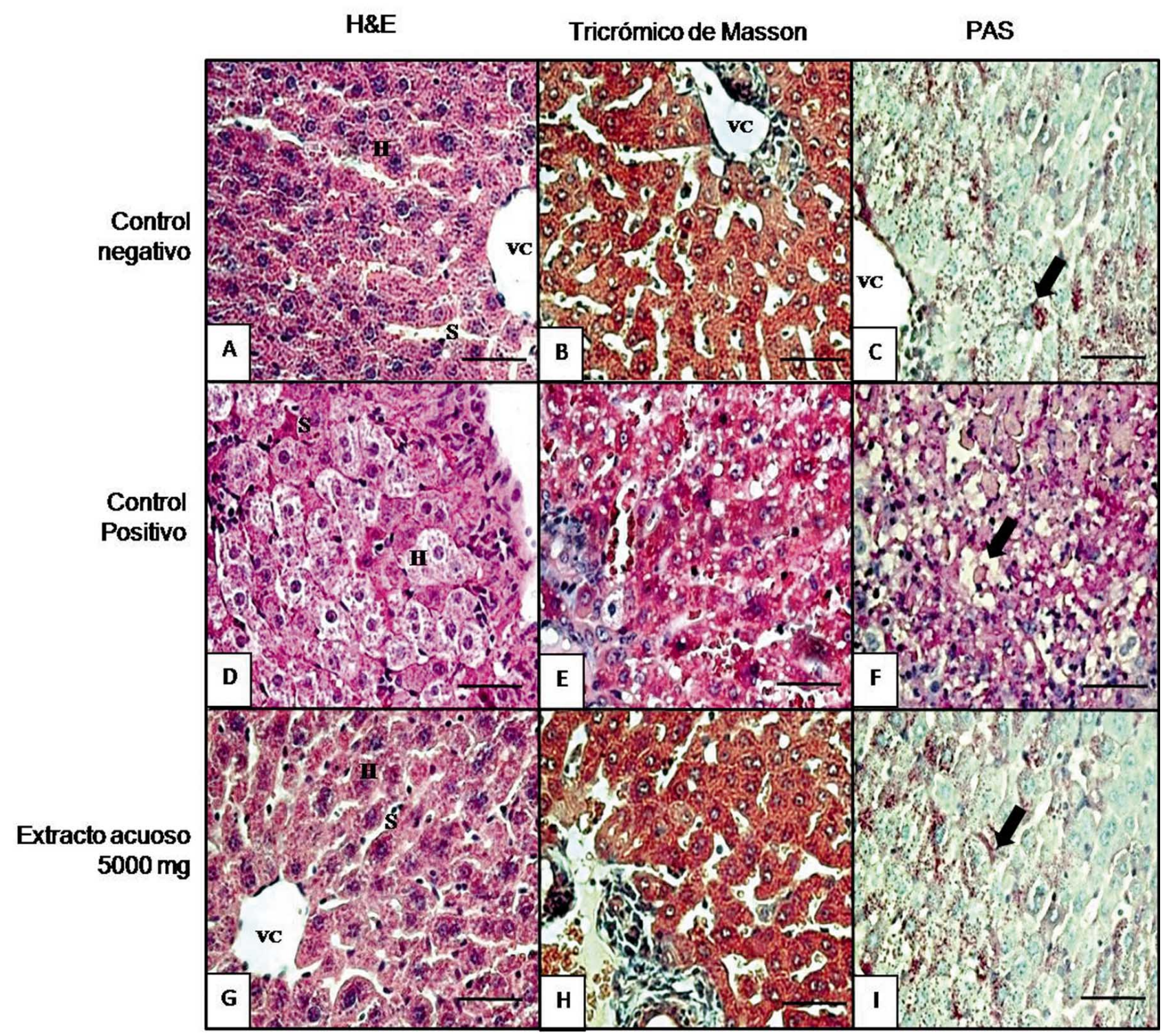

Fig. 1. Grupo control negativo: A) Hepatocitos (H), sinusoides (S) y vena central (VC) de características histológicas normales. B): Muestra los mismos hallazgos descritos para A. C) Se observan pequeños depósitos de glucógeno de aspecto granulado en los hepatocitos (flecha). Grupo control positivo: D) Presencia de necrosis hepática $(\mathrm{H})$ además de congestión sinusoidal (S). E): Muestra los mismos hallazgos descritos para D. F) se observan zonas con material PAS positivo en el espacio extracelular, además de una evidente destrucción de los mismos (flecha). Grupo tratado con 5000 mg de extracto acuoso de orégano: G) Hepatocitos (H), sinusoides (S) y vena central (VC) de características histológicas normales. H): Muestra los mismos hallazgos descritos para G. I) Se observan pequeños depósitos de glucógeno de aspecto granulado en los hepatocitos (flecha). Microscopia de luz, inclusión en parafina. A, B y C: tinción de H\&E. D,E y F: tinción de tricrómico de Masson. G, H e I: reacción de PAS. Barra $50 \mu$ m.

colectores con características histológicas normales (Fig. 2 A y B). Con la técnica de PAS se observaron microvellosidades íntegras con positividad en el glucocáliz (Fig. 2C). Esto contrasta con lo observado en el grupo control positivo, cuyas porciones tubulares de la corteza (Fig. 2D) y médula (Fig. 2E) muestran extensas zonas con signos de necrosis, caracterizada por ruptura de la membrana plasmática, extracción total o parcial del citoplasma y núcleos con pérdida de la cromatina, también se observó congestión vascular con zonas de hemorragia y retracción de los capilares glomerulares. Al analizar los cortes con la técnica de PAS, se observaron células epiteliales con destrucción de las microvellosidades (Fig. 2F). Destaca que en los grupos tratados con diferentes concentraciones del extracto de orégano se observó una histología normal similar al control negativo (Fig. $2 \mathrm{G}, \mathrm{He}$ I).

Los resultados del análisis cuantitativo del riñón en el grupo control positivo muestran una notable disminución de túbulos proximales y distales normales $(51,83 \pm 0,56)$, mientras que en los grupos control negativo y tratados con 


\section{Tricrómico de Masson}

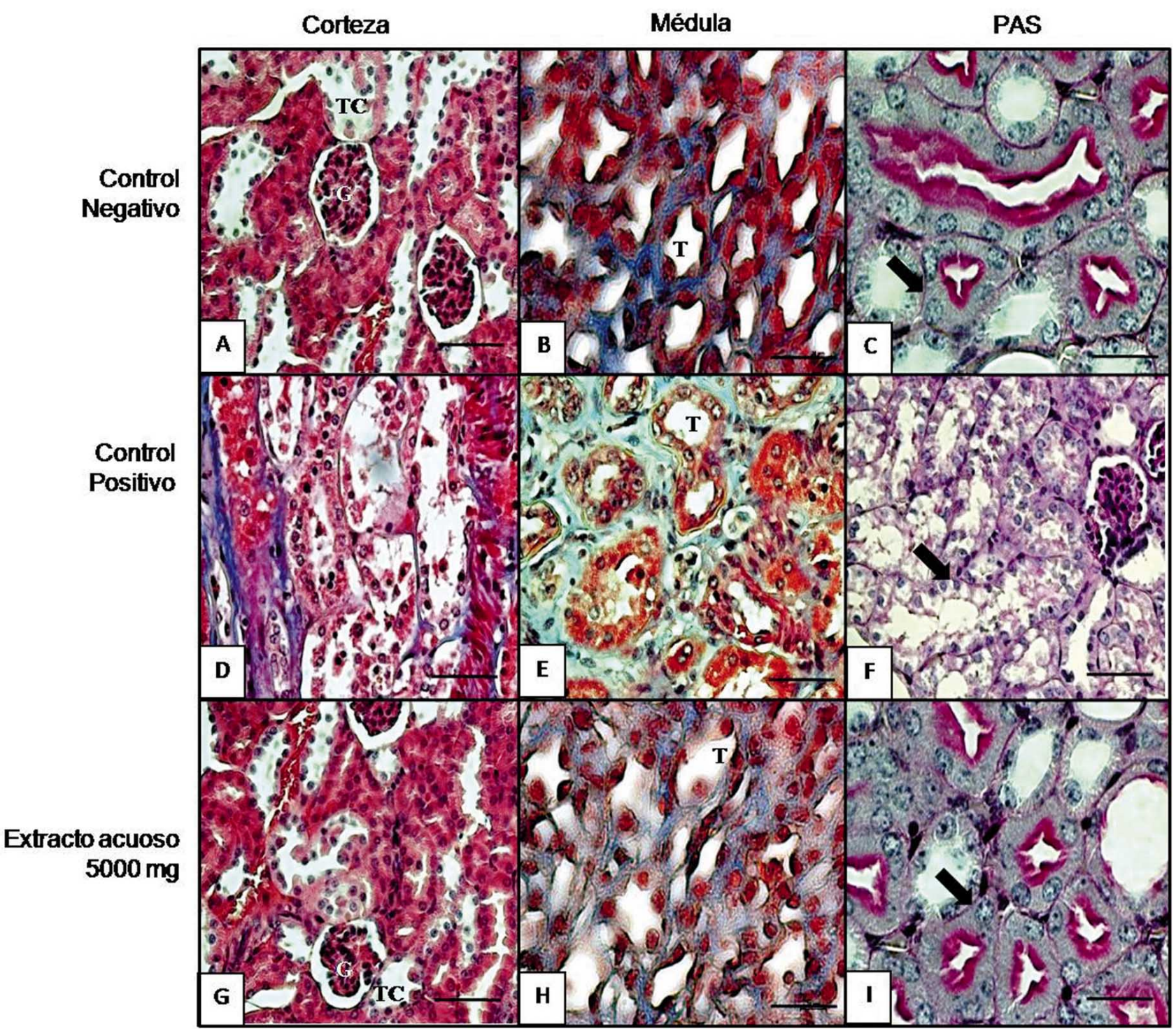

Fig. 2. Grupo control negativo: A) Corteza renal que muestra la presencia de glomérulos renales (G) y túbulos contoneados (TC) de características histológicas normales. B): Médula renal que muestra a los túbulos (T) tanto colectores como asas de Henle de características histológicas normales. C) Membranas basales delgadas y continuas sin alteraciones (flecha). Grupo control positivo: D) Presencia de necrosis tubular en los túbulos contorneados (t). E): Destrucción tubular (T) a nivel medular. F) Pérdida de la continuidad de la membrana basal de los túbulos (flecha). Grupo tratado con $5000 \mathrm{mg}$ del extracto acuoso de orégano: G) Corteza renal que muestra la presencia de glomérulos renales $(\mathrm{G})$ y túbulos contoneados (TC) de características histológicas normales. H): Medula renal que muestra a los túbulos de características histológicas normales. I) Membranas basales delgadas y continuas sin alteraciones (flecha). Microscopia de luz inclusión en parafina. A, B y C: tinción de H\&E. D, E y F: tinción de tricrómico de Masson. G,H e I: reacción de PAS. Barra 50 m.

Tabla I. Porcentaje de hepatocitos normales.

\begin{tabular}{|c|c|c|c|c|c|c|}
\hline \multirow[b]{2}{*}{ GRUPO } & \multicolumn{2}{|c|}{ Zona 1} & \multicolumn{2}{|c|}{ Zona 2} & \multicolumn{2}{|c|}{ Zona 3} \\
\hline & PROMEDIO & DE & PROMEDIO & DE & PROMEDIO & DE \\
\hline C. Negativo & 99,91 & $\pm 0,12$ & 100 & \pm 0 & 99,66 & $\pm 0,34$ \\
\hline C. Positivo & $65,41 *$ & $\pm 4,85$ & $60,58 *$ & $\pm 4,49$ & $58,41 *$ & $\pm 9,05$ \\
\hline $5,000 \mathrm{mg}$ & 99,08 & $\pm 1,02$ & 99,58 & $\pm 0,64$ & 99,41 & $\pm 0,56$ \\
\hline $1.000 \mathrm{mg}$ & 99,66 & $\pm 0,34$ & 99,58 & $\pm 0,64$ & 99,58 & $\pm 0,12$ \\
\hline $100 \mathrm{mg}$ & 99,50 & $\pm 0,59$ & 99,41 & $\pm 0,56$ & 99,75 & $\pm 0,22$ \\
\hline $10 \mathrm{mg}$ & 99,75 & $\pm 0,22$ & 99,91 & $\pm 0,12$ & 99,75 & $\pm 0,22$ \\
\hline
\end{tabular}

$* \mathrm{p} \leq 0,05$ 
Tabla II. Porcentaje de túbulos renales normales.

\begin{tabular}{lcc}
\hline GRUPO & PROMEDIO & DE \\
\hline C. Negativo & 98,33 & $\pm 0,12$ \\
C. Positivo & $51,83 *$ & $\pm 0,56$ \\
$5.000 \mathrm{mg}$ & 96,25 & $\pm 2,15$ \\
$1.000 \mathrm{mg}$ & 98,40 & $\pm 0,20$ \\
$100 \mathrm{mg}$ & 98,52 & $\pm 0,59$ \\
$10 \mathrm{mg}$ & 98,51 & $\pm 0,25$ \\
\hline
\end{tabular}

el extracto de orégano se obtuvieron valores de $98,33 \pm 0,12$ y $96,25 \pm 2,15$ para la mayor concentración respectivamente (Tabla II).

El efecto morfológico no tóxico observado en este estudio con el extracto acuoso de Lippia graveolens HBK, se correlaciona con lo descrito en la literatura sobre el extenso uso del orégano como analgésico; contra diarrea, vómito, indigestión y disentería (Monroy \& Castillo); antialérgico, antiviral, vasodilatador y antihipertensivo (Guerrero et al.), antifúngico (Hernández et al., 2008), antiparasitario (Bassole et al.); antígenotóxico (Hirobe et al.); antimicrobiano (Pascual et al.; Hernández et al., 2009); espasmolítico y estrogénico (Pérez); en el tratamiento de desórdenes hepáticos, sífilis, gonorrea y diabetes (Socorro et al.; Pascual et al.; ArcilaLozano et al.). También se ha descrito su uso contra bacterias gram negativas, gram positivas (Hernández et al., 2009) y fungicida (Sivropoulou et al.). Por lo que nuestros resultados demuestran que al utilizar los extractos acuosos de Lippia graveolens $\mathrm{HBK}$ en el tratamiento de las patologías descritas, no se induce un daño celular o efecto tóxico sobre órgano importantes como hígado y riñón.

Los resultados obtenidos en este estudio contribuyen de manera importante en la investigación de los efectos fitoterapéuticos, farmacológicos y toxicológicos de las plantas del genero Lippia, además de demostrar la actividad antioxidante y los efectos morfológicos in vitro e in vivo de un extracto acuoso de Lippia graveolens HBK. Actualmente en nuestro laboratorio se llevan a cabo estudios para identificar los compuestos químicos presentes en el extracto acuoso evaluado y/o extractos metanólicos y aceites esenciales de las plantas del género Lippia de esta región de México, para la posterior evaluación de sus efectos sobre modelos in vitro e in vivo.

\section{AGRADECIMIENTOS}

Yolanda Ramírez Casas agradece al Consejo Nacional de Ciencia y Tecnología (CONACYT), por el apoyo económico brindado con la beca 216337, para la realización de sus estudios de Maestría en Ciencias en Investigación Multidisciplinaria de Salud.

SOTO-DOMÍNGUEZ, A.; GARCÍA-GARZA, R.; RAMÍREZ-CASAS, Y.; MORÁN-MARTÍNEZ, J. \& SERRANO-GALLARDO, L. B. The aqueous extract from oregano (Lippia graveolens HBK) from the North of Mexico has antioxidant activity without showing a toxic effect in vitro e in vivo. Int. J. Morphol., 30(3):937-944, 2012.

SUMMARY: Since ancient times, traditional medicine has widely used species of the genus Lippia as analgesics, anti-inflammatory, antipyretic, antifungal, etc. Numerous studies describe several compounds present in aqueous extracts, methanol, or essential oils of these plants, being flavonoids the most abundant compounds. However, the composition and quantity of secondary metabolites depend on the geographical area, climatic factors, altitude, time of harvest and growth status of these plants. The objective of this study was to evaluate the antioxidant activity of aqueous extract of oregano (Lippia graveolens $H B K$ ) from the North of Mexico and its toxic effect in vitro and in vivo. The antioxidant activity of the aqueous extract was measured by DPPH method in six dilutions of the extract (5-160 mg $/ \mathrm{mL}$ ), Trolox was used as a reference. For the in vitro toxic effect, cytotoxicity assay with larvae of Artemia salina was used. For the in vivo effect, 24 males mice were used and divided into 6 groups $(\mathrm{n}=4): 4$ experimental groups with 10, 100, 1000 and 5000 mg extract / $\mathrm{kg}$ body weight administered orally respectively, also we used a group positive control ( $5 \mathrm{mg}$ of colchicine / $\mathrm{kg}$ body weight administered via ip) and a negative control group (distilled water only). After treatment all mice were sacrificed, and samples from liver and kidney were collected and analyzed by histological and histochemical studies. Also a quantitative analysis was done. The antioxidant activity of aqueous extract of oregano was presented at $160 \mathrm{mg} / \mathrm{mL}$. The LC50 was greater than $1.000 \mathrm{mg} / \mathrm{mL}$, so the extract is considered nontoxic. In the morphological analysis in vivo with different doses of aqueous extract of oregano, no toxic effect was observed. The results validate the use of oregano in traditional medicine.

KEY WORDS: Antioxidant; Oregano; Morphological analysis.

\section{REFERENCIAS BIBLIOGRAFICAS}

Arcila-Lozano, C. C.; Loarca-Piña, G.; Lecona-Uribe, S. \& González de Mejía, E. El orégano: propiedades, com- posición y actividad biológica de sus componentes. Arch. Lat. Nut., 54:100-11, 2004. 
SOTO-DOMÍNGUEZ, A.; GARCÍA-GARZA, R.; RAMÍREZ-CASAS, Y.; MORÁN-MARTÍNEZ, J. \& SERRANO-GALLARDO, L. B. El extracto acuoso de oregano (Lippia graveolens HBK) del norte de México tiene actividad antioxidante sin mostrar un efecto toxico in vitro e in vivo. Int. J. Morphol., 30(3):937-944, 2012.

Bassole, I. H.; Ouattara, A. S.; Nebie, R.; Ouattara, C. A.; Kabore, Z. I. \& Traore, S. A. Chemical composition and antibacterial activities of the essential oils of Lippia chevalieri and Lippiamultiflora from Burkina Faso. Phytochem, 62:209-12, 2003.

Burt, S. A. \& Reinders, R. D. Antibacterial activity of selected plant essential oils against Escherichia coli O157:H7. Lett. Appl. Microbiol., 36:162-7, 2003.

Cimanga, K.; Kambu, K.; Tona, L.; Apers, S.; De Bruyne, T.; Hermans, N.; Totté, J.; Pieters, L. \& Vlietinck, A. J. Correlation between chemical composition and antibacterial activity of essential oils of some aromatic medicinal plants growing in the Democratic Republic of Congo. J. Ethnopharmacol., 79:213-20, 2002.

Craker, L. \& Simon, J. E. Herbs, Spices and Medicinal Plants. Recent Advances in Botany, Horticulture and Pharmacology. V. 3. New York, Food Products Press, 1992. pp.103-44.

de las Heras, B.; Slowing, K.; Benedí, G.; Carretero, E.; Ortega, T.; Toledo, C.; Bermejo, P.; Iglesias, I.; Abad, M. J.; Gómez-Serranillos, P.; Liso, P. A.; Villar, A. \& Chiriboga, X. Antinnflammatory and antioxidant activity of plants used in traditional medicine in Ecuador. $J$. Ethnopharm., 61:161-6, 1998.

Dragland, S.; Senoo, H.; Wake, K.; Holte, K. \& Blomhoff, R. Several culinary and medicinal herbs are important sources of dietary antioxidants. J. Nutr., 133:1286-90, 2003.

González-Güereca, M. C.; Soto-Hernández, M.; Kite, G. \& Martínez-Vázquez, M. Actividad antioxidante de flavonoides del tallo de orégano mexicano (Lippia graveolens HBK var. berlandieri schauer). Rev. Fitotec. Mex., 30:43-9, 2007.

Guerrero, M. F.; Puebla, P.; Carrón, R.; Martín, M. L.; Arteaga, L. \& San Román, L. Assessment of the antihypertensive and vasodilator effects of ethanolic extracts of some Colombian medicinal plants. $J$. Ethnopharm., 80:37-42, 2002.

He, L.; Mo, H.; Hadisusilo, S.; Quresh, A. A. \& Elson, C. E. Isoprenoids suppress the growth of murine B16 melanomas in vitro and in vivo. J. Nutr., 127:668-73, 1997.

Hernández, T.; Canales, M.; Ávila, J. G.; García, A. M.; Meraz, S. \& Duran, A. Antifungal activity of the essential oils of two Verbenaceae: Lantana achyranthifolia Desf.
And Lippia graveolens H.B.K. of Zapotitlán de las Salinas, Puebla (México). Bol. Latinoamer. Caribe Plant. Med. Aromat., 7:203-7, 2008.

Hernández, T.; Canales, M.; Ávila, J. G.; García, A. M.; Meraz, S.; Caballero, J. \& Lira, R. Composition and antibacterial activity of essential oil of Lippia graveolens H.B.K. (Verbenaceae). Bol. Latinoam. Caribe Plant. Med. Aromat., 8:295-300, 2009.

Hirobe, C.; Qiao, Z. S.; Takeya, K. \& Ibokawa, H. Cytotoxic principles from Majorama syriaca. Nat. Med., 52:74-7, 1998.

Huerta, C. Orégano mexicano: oro vegetal. Biodiversitas, 3:8-13, 1997.

Lecona-Uribe, S.; Loarca-Piña, F. G.; Arcila-Lozano, C.; Díaz-Moscoso, C. \& Ocampo, R. Nutraceutical potential of Mexican oregano (Lippia graveolens K). IFT Annual Meeting, 14E-28, 2003.

Meyer, B. N.; Ferrigni, N. R.; Putnam, J. E., Jacobsen, L. B., Nichols, D. E. \& Melaughlin, J. L. Brine shrimp: A convenient general bioassay for active plant constituents. Planta Med., 45:31-4, 1982.

Monroy, C. \& Castillo, P. Plantas medicinales utilizadas en el estado de Morelos, Centro de Investigaciones Biológicas. Morelos, Universidad Autónoma del Estado de Morelos, 2000.

Ohnishi, M.; Morishita, H.; Iwahashi, H.; Toda, S.; Shirataki, Y.; Kimura, M. \& Kido, R. Inhibitory effects of chlorogenic acids on linoleic acid peroxidation and haemolysis. Phytochemistry, 36:579-83, 1994.

Pascual, M. E.; Slowing, K.; Carretero, E.; Sánchez, M. D. \& Villar, A. Lippia: Traditional uses, chemistry and pharmacology: A review. J. Ethnopharmacol., 76:20114, 2001.

Pérez, G. R. M. Compuestos Aislados de Plantas con Actividad Antiinflamatoria, Antiviral e Hipoglucemiante. México D.F., Instituto Politécnico Nacional, 2002.

Pierce, A. Practical guide to natural medicines. The American Pharmaceutical Association. A Stonesong Press Book. New York, William Morrow and Company Inc., 1999. pp.728.

Pietta, P. G. Flavonoids as Antioxidants. J. Nat. Prod., 63:1035-42, 2000. 
SOTO-DOMÍNGUEZ, A.; GARCÍA-GARZA, R.; RAMÍREZ-CASAS, Y.; MORÁN-MARTÍNEZ, J. \& SERRANO-GALLARDO, L. B. El extracto acuoso de oregano (Lippia graveolens HBK) del norte de México tiene actividad antioxidante sin mostrar un efecto toxico in vitro e in vivo. Int. J. Morphol., 30(3):937-944, 2012.

Ricco, R. A.; Warner, M. L.; Portmann, E.; Reides, C.; Llesuy, S.; Gurni, A. A. \& Carballo, M. A. Análisis de polifenoles, actividad antioxidante y genoytoxicidad en especies argentinas de Lippia y Aloysia (Verbenaceae). Bol. Latinoam. Caribe Plant. Med. Aromat., 9:388-96, 2010.

Rzedowski, J. Vegetación de México. México D.F., Limusa, 1978. pp.432.

Salgueiro, L. R.; Cavaleiro, C.; Goncalves, M. J. \& Proenca da Cunha, A. Antimicrobial activity and chemical composition of the essential oil of Lippia graveolens from Guatemala. Planta Med., 69:80-3, 2003.

Salinas-Sánchez, D. O.; Arteaga-Nájera, G. L.; León-Rivera, I.; Dorado-Ramírez, O.; Valladares-Cisneros, M. G. \& Navarro-García, V. M. Actividad antimicrobiana de las plantas medicinales de la reserva de la biosfera Sierra de Huautla en Morelos (México). Polibotánica, 28:213-25, 2009.

Sivropoulous, A.; Papanikolaou, E.; Nikolaou, C.; Kokkini, S.; Lanaras, T. \& Arsenakis, M. Antimicrobial and cytotoxic activities of Origanum essential oils. J. Agric. Food Chem., 44:1202-5, 1996.

Skandamis, P.; Tsigarida, E. \& Nychas, G-J. E. The effect of oregano essential oil on survival/death of Salmonella typhimurium in meat stored at $5^{\circ} \mathrm{C}$ under aerobic, VP/ MAP conditions. Food Microbiol., 19:97-103, 2002.

Tárrega, I. \& Rivas, F. Essential oils from wild and micropropagated plants of Origanum bastetanum. Phytochem., 48:1347-9, 1998.

Valentão, P.; Fernández, E.; Carvalho, F.; Andrade, P. B.; Seabra, R. M. \& Bastos, M. L. Studies on the antioxidant activity of Lippia citriodora infusion: scavenging effect on superoxide radical, hydroxyl radical and hypochlorous acid. Biol. Pharm. Bull., 25:1324-7, 2002.

Zavala-Nigoa, J.; Loarca-Piña, G. \& García-Gasca, T. Evaluación del contenido fenólico, capacidad antioxidante y actividad citotóxica sobre células $\mathrm{CaCo-2}$ del extracto acuoso de orégano (Lippia graveolens kunth). Querétaro, México, 4 al 8 de septiembre, $2^{\circ}$ Congreso Nacional de Química Médica, 2006.

Zétola, M.; De Lima, T. C.; Sonaglio, D.; González-Ortega, G.; Limberger, R. P.; Petrovick, P. R. \& Bassani, V. L. CNS activities of liquid and spray-dried extracts from Lippia alba-Verbenaceae (Brazilian false melissa). $J$. Ethnopharmacol., 82:207-15, 2002.
Dirección para correspondencia:

Adolfo Soto-Domínguez

Departamento de Histología

Facultad de Medicina

Universidad Autónoma de Nuevo León

MÉXICO

Email: ibqasoto@yahoo.com.mx

Recibido : 03-11-2011

Aceptado: 07-07-2012 